The authors' regional IgG4-RD service is one of the largest UK-based units treating patients with this condition. Specialist clinics and multidisciplinary team meetings operate alongside an active research programme. We aimed to describe the frequency with which thoracic abnormalities - either as a symptomatic presenting feature of IgG4-RD or an incidental asymptomatic finding on imaging - were present in a prospectively recruited patient cohort.

Method and results Patients referred to the authors' IgG4-RD service from 2005 onwards and confirmed as having a diagnosis of IgG4-RD were included. Diagnoses were made using established clinical criteria (HISORt for AIP and Japanese International Consensus Diagnostic Criteria for systemic disease); tissue specimens were assessed using the Boston histopathological consensus criteria where available. Patients were followed prospectively; clinicopathological data relating to presentation and clinical progress were stored in a secure database with the consent of participants. In patients without symptomatic thoracic manifestations of IgG4-RD, routine clinical imaging (CXR and CT) was reviewed where available for evidence of incidental asymptomatic disease.

61 IgG4-RD patients with thoracic imaging available were included; mean age at diagnosis was 60.3 years (SD 14.6). 43 $(70.5 \%)$ patients were male. The majority of patients $(85.2 \%)$ presented with features of intra-abdominal disease. 6 patients (9.8\%) had evidence of symptomatic thoracic disease on the basis of clinical presentation, radiology and/or histology. A further $15(24.6 \%)$ patients had abnormal imaging suggestive of asymptomatic thoracic IgG4-RD.

Conclusion A significant proportion of IgG4-RD patients have evidence of symptomatic and asymptomatic thoracic manifestations of this multi-system disease. Respiratory physicians should consider IgG4-RD in their differential diagnosis for a range of pulmonary presentations, particularly where there is co-existing extra-thoracic organ involvement. Making a diagnosis of IgG4RD impacts on access to established therapeutic options including corticosteroids and rituximab to which the disease is responsive in the inflammatory phase.

\section{P208 PREVENTION OF VENTILATOR-ASSOCIATED PNEUMONIA WITH CHEST PHYSIOTHERAPY: A META-ANALYSIS}

GF Javelosa, RK De Borja, M Lagmay, K Villareal. University of the East Ramon Magsaysay Memorial Medical Center, Quezon City, Philippines

10.1136/thoraxjnl-2015-207770.344

Research question Among mechanically ventilated adult ICU patients, can chest physiotherapy (CPT) prevent the onset of ventilator-associated pneumonia (VAP) compared with standard care?

Introduction VAP is a common nosocomial infection with various known strategies for prevention, including CPT. Conflicting evidence regarding CPT for VAP prevention exist since CPT may cause desaturation and respiratory muscle fatigue.

Objectives To determine the efficacy of CPT, compared with standard care, in preventing the onset of VAP among mechanically ventilated adult ICU patients, its effect on ICU mortality, length of ICU stay, and duration of mechanical ventilation.

Inclusion criteria Controlled trials on adult mechanically ventilated ICU patients, given CPT for VAP prevention, compared with standard care.

Search strategy An electronic search in PubMed, EMBASE, CENTRAL, BioMedCentral, Elsevier Health, and Herdin was done. Reference lists were checked manually.

Study manoeuvres The authors arrived at a consensus and the Cochrane risk of bias tool was used for evaluation.

Statistical analysis Mantel-Haenszel method using the Review manager 5.3.

Results Twenty studies were found, and 10 were retrieved for review. Five studies were included, representing 595 patients. Evaluation of the included studies found 1 study with low risk of bias, 2 studies with high risk, and 2 studies with unclear risk. Overall combined meta-analysis of all 5 studies found no difference in VAP incidence between the 2 groups (RR 0.80, 95\% CI 0.52 to $1.23, \mathrm{P}=0.05)$. A subgroup analysis done excluding the studies with high risk of bias still showed no difference in VAP incidence ( $R R 0.96,95 \%$ CI 0.62 to $1.50, P=0.86$ ). CPT made no significant difference on ICU mortality (RR 0.97, 95\% CI 0.57 to $1.97, \mathrm{P}=0.07$ ), duration of ICU stay (RR 0.36, $95 \% \mathrm{CI}-1.83$ to $2.55, \mathrm{P}=0.10$ ), and duration of mechanical ventilation (RR $0.23,95 \% \mathrm{CI}-0.74$ to $1.21, \mathrm{P}=0.14$ ).

Conclusions It is not recommended to perform routine CPT on mechanically ventilated adult ICU patients to prevent the onset of VAP, as this is associated with potential harm and unnecessary costs. The authors recommend that more trials with low risk of bias be conducted on CPT for VAP prevention.

\section{P209 THE BURDEN OF HOSPITAL ACQUIRED PNEUMONIA: A COHORT STUDY}

${ }^{1} V$ Navaratnam, ${ }^{1} \mathrm{E}$ O'Dowd, ${ }^{2} \mathrm{H}$ Staniforth, ${ }^{3} \mathrm{~L}$ Haynes, ${ }^{2} \mathrm{~J}$ Lacey, ${ }^{4} \mathrm{~A}$ Gummow, ${ }^{1}$ RB Hubbard, ${ }^{5} \mathrm{~T}$ Bewick. ${ }^{1}$ Division of Epidemiology and Public Health, University of Nottingham, Nottingham, UK; ${ }^{2}$ Department of Pharmacy, Derby Teaching Hospitals NHS Foundation Trust, Derby, UK; ${ }^{3}$ Department of Clinical Coding, Derby Teaching Hospitals NHS Foundation Trust, Derby, UK; ${ }^{4}$ Department of Radiology, Derby Teaching Hospitals NHS Foundation Trust, Derby, UK; ${ }^{5}$ Department of Respiratory Medicine, Derby Teaching Hospitals Foundation Trust, Derby, UK

10.1136/thoraxjnl-2015-207770.345

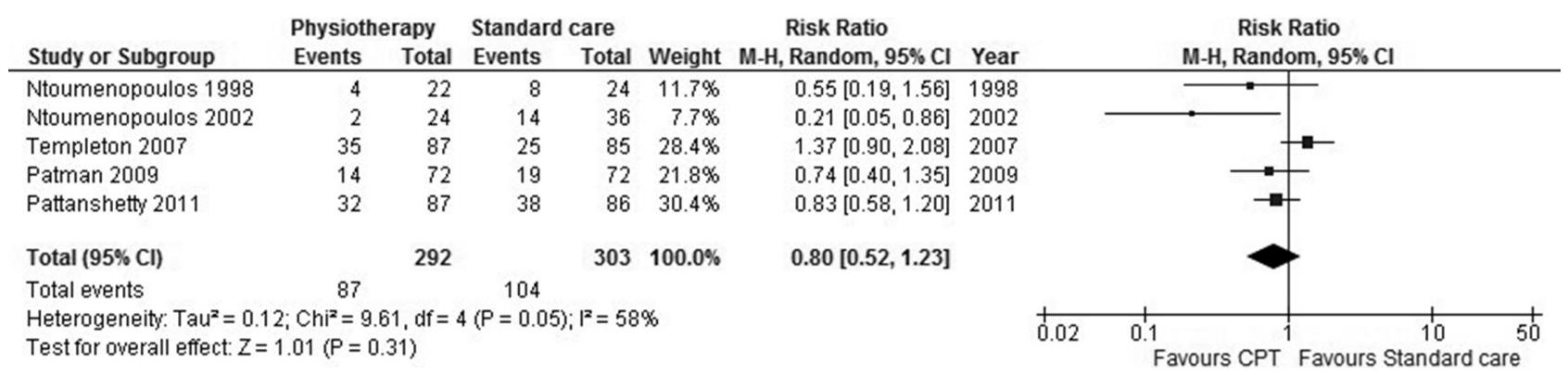

Abstract P208 Figure 1 